VISCERAL SENSATION

\title{
Different contributions of ASIC channels 1a, 2, and 3 in gastrointestinal mechanosensory function
}

\author{
A J Page*, S M Brierley*, C M Martin*, M P Price, E Symonds, R Butler, J A Wemmie, L A Blackshaw
}

Gut 2005;54:1408-1415. doi: 10.1136/gut.2005.071084

See end of article for authors' affiliations

Correspondence to: Dr L A Blackshaw, Nerve Gut Research Laboratory, Hanson Institute, Frome Road, Adelaide SA5000 Australia; ablacksh@mail. rah.sa.gov.au

Accepted 8 June 2005

\begin{abstract}
Aims: Members of the acid sensing ion channel (ASIC) family are strong candidates as mechanical transducers in sensory function. The authors have shown that ASICla has no role in skin but a clear influence in gastrointestinal mechanotransduction. Here they investigate further $\mathrm{ASICla}$ in gut mechanoreceptors, and compare its influence with ASIC2 and ASIC3.

Methods and results: Expression of $\mathrm{ASICla}, 2$, and 3 mRNA was found in vagal (nodose) and dorsal root ganglia (DRG), and was lost in mice lacking the respective genes. Recordings of different classes of splanchnic colonic afferents and vagal gastro-oesophageal afferents revealed that disruption of ASICla increased the mechanical sensitivity of all afferents in both locations. Disruption of ASIC2 had varied effects: increased mechanosensitivity in gastro-oesophageal mucosal endings, decreases in gastrooesophageal tension receptors, increases in colonic serosal endings, and no change in colonic mesenteric endings. In ASIC3-/- mice, all afferent classes had markedly reduced mechanosensitivity except gastrooesophageal mucosal receptors. Observations of gastric emptying and faecal output confirmed that increases in mechanosensitivity translate to changes in digestive function in conscious animals.

Conclusions: These data show that ASIC3 makes a critical positive contribution to mechanosensitivity in three out of four classes of visceral afferents. The presence of ASICla appears to provide an inhibitory contribution to the ion channel complex, whereas the role of ASIC2 differs widely across subclasses of afferents. These findings contrast sharply with the effects of $A S I C l, 2$, and 3 in skin, suggesting that targeting these subunits with pharmacological agents may have different and more pronounced effects on mechanosensitivity in the viscera.
\end{abstract}

W have recently shown in mice that there are two major populations of mechanoreceptor in the vagal innervation of the stomach and oesophagus that signal muscle stretch and mucosal stroking, ${ }^{1}$ and two major populations in the splanchnic innervation of the colon that signal distortion of the serosa and mesentery. ${ }^{2}$ These populations subserve roles not only in behaviour, including food intake and pain, but also in reflex regulation of digestion.

Understanding the molecular basis of mechanotransduction is currently a major challenge. Mechanotransduction is fundamental to the senses of hearing, touch, proprioception, and various aspects of interoception (sensing from internal organs). The number of candidate molecules as mechanotransducers is increasing with the discovery of novel molecules and improved understanding of established molecules. The major candidates in touch sensation are two families of ion channels: the degenerin/epithelial sodium channel (DEG/ENaC) family, and the transient receptor potential (TRP) family. The DEG/ENaCs in mammals are comprised mainly of the acid sensing ion channels (ASICs). ${ }^{3}{ }^{4}$ Candidate TRP channels in mechanotransduction are TRPVI, $\mathrm{V} 4$, and Al. ${ }^{5-7}$ ASICs have been localised to peripheral mechanosensory structures and sensory ganglia. ${ }^{8-14}$ They were implicated in mechanotransduction by their close relation to invertebrate channels, without which there are major deficits in body touch perception. ${ }^{3415}$ A more direct implication in mechanotransduction came from studies of five classes of cutaneous mechanoreceptors in mutant mice lacking individual ASIC family members. These are: rapidly adapting (RA) and slowly adapting (SA) mechanoreceptors; down hair (D-hair) receptors; A-fibre mechanonociceptors (AM), and high threshold C-fibres. Disruption of ASIC2 (also known as $B N C l$ ) reduced responses of RA and SA mechanoreceptors. ${ }^{11}$ Disruption of ASIC3 (or DRASIC) reduced the responsiveness of AM nociceptors, whereas (RA) mechanoreceptors in contrast showed increased mechanosensitivity. ${ }^{12}$ The $A S I C I$ gene (also known as $A S I C$ ) gives rise to two proteins (ASICla and $\mathrm{lb}$ ) through alternative splicing. Disruption of $A S I C l$ using the currently available strategy leads to loss of transcript only for ASICla, but expression of both ASICla and $1 \mathrm{~b}$ proteins is successfully abolished..$^{10}$ This knockout is therefore referred to henceforth specifically as ASICla -/-. This knockout had no effect on any cutaneous mechanoreceptors. ${ }^{10}$ The first investigation of these ion channels in visceral afferents showed that ASICla contributes to gastro-oesophageal and colonic afferent mechanotransduction, ${ }^{10}$ with an increase in mechanosensitivity after ASICla disruption. It is clear from knockout, patch clamp, and expression studies that ASICla, 2, and 3 coexist in the same sensory neurones, and that they form functional heteromultimers. ${ }^{316}$ The picture that emerges from these positive and negative effects of ASIC mutation is of a complex interplay between individual members of the heteromultimer, which explains the positive and negative effects of disruption of each member.

Here we tested the hypothesis that ASICs contribute to visceral mechanoreceptors by studying mice bearing targetted disruptions of the ASIC genes. We studied different aspects of mechanosensitivity in different classes of mechanoreceptor throughout the gut. The physiological relevance of changes in mechanoreceptor function was assessed by observing altered digestive activity in conscious animals with or without ASIC genes.

Abbreviations: ASIC, acid sensing ion channel; DRG, dorsal root ganglion/ganglia; TRP, transient receptor potential. 
Table 1 Primers used for RT-PCR identification of ASIC transcripts in nodose and dorsal root ganglia

\begin{tabular}{lll}
\hline & & Sequence 5'-3' \\
\hline ASIC1 & Forward & CAGCTAGAGATATGGCAGGACAAG \\
& Reverse & CCACACAGGCAAGTACTATCTTG \\
ASIC2 & Forward & TTCGAGAACATCTTGTCTGGAT \\
& Reverse & GTCTCATCATGGCTCCCTCCTC \\
AS IC3 & Forward & TGAGAGCCACCAGCTIACC \\
& Reverse & GGCAGATACTCCTCCTGCT \\
$\beta$-actin & Forward & ATCATGTTGAGACCTCAACAC \\
& Reverse & TCTGCGCAAGTAGGTTTGTC \\
\hline
\end{tabular}

$\mathrm{ASICl}$ and 2 primer pairs were as previously used."

\section{METHODS}

\section{Generation of ASIC null mutant mice}

Mice with disrupted ASIC genes were generated by homologous recombination in embryonic stem cells as we have previously described. ${ }^{11}{ }^{12} 17$ Heterozygote pairs of mice were mated to generate animals for the current investigation. Homozygous ASICla, 2, and 3+/+ or -/- littermates were blindly selected for experiments after genotyping and results were encoded until statistical analysis. Littermates were otherwise indistinguishable by body weight, general behaviour, or gross anatomical features of the gut or elsewhere.

\section{Determination of ASIC expression}

RNA was isolated from nodose ganglia and lumbar dorsal root ganglia (DRG) from ASICla, 2, and 3+/+ and -/- mutant mice using homogenisation and TRIzol reagent (Invitrogen, Australia), followed by isopropanol precipitation. Reverse transcription (RT) and polymerase chain reaction (PCR) was performed using a Qiagen one-step RT-PCR kit, with primers used as indicated in table 1 under the following conditions: reverse transcription: $50^{\circ} \mathrm{C}$ for 30 minutes; initial PCR activation: $95^{\circ} \mathrm{C}$ for 15 minutes; PCR cycles: $94^{\circ} \mathrm{C}$ for 1 minute, $48^{\circ} \mathrm{C}$ for 1 minute, $72^{\circ} \mathrm{C}$ for 1 minute repeated for 40 cycles. Control PCRs were performed by substituting RNA template with distilled water. Amplified products were resolved by $1.5-3 \%$ agarose gel electrophoresis and visualised via ethidium bromide staining.

\section{Electrophysiology}

In vitro colonic lumbar splanchnic nerve recording Using an approach established in rat $\operatorname{colon}^{18}$ and adapted recently for mouse, ${ }^{2}$ the distal colon and attached lumbar splanchnic nerves were removed from ASICla, 2, and $3+/+$ and -/- mutant mice. The colon was opened and pinned flat mucosal side up in a specialised organ bath. The colonic compartment was superfused with a modified Krebs solution of the following composition (mM): $117.9 \mathrm{NaCl}, 4.7 \mathrm{KCl}, 25$ $\mathrm{NaHCO}_{3}, 1.3 \mathrm{NaH}_{2} \mathrm{PO}_{4}, 1.2 \mathrm{MgSO}_{4}\left(\mathrm{H}_{2} \mathrm{O}\right)_{7}, 2.5 \mathrm{CaCl}_{2}, 11.1$
D-Glucose, 2 NaButyrate, and 20 NaAcetate bubbled with carbogen $\left(95 \% \mathrm{O}_{2} / 5 \% \mathrm{CO}_{2}\right)$ at $34^{\circ} \mathrm{C}$. In all preparations the Ltype calcium channel antagonist nifedipine $(1 \mu \mathrm{M})$ was added to suppress smooth muscle activity and the prostaglandin synthesis inhibitor indomethacin $(3 \mu \mathrm{M})$ added to suppress potential inhibitory actions of endogenous prostaglandins. The nerve bundle was extended into the paraffin filled recording compartment where after fine dissection strands were laid onto a mirror and single fibres recorded. ${ }^{2}{ }^{10}$

Receptive fields were identified as described previously. ${ }^{2}$ Serosal and mesenteric afferents were chosen rather than muscular or mucosal afferents based on their relative abundance. $^{2}$ Serosal afferents were located on the colonic wall, whereas mesenteric afferents were distributed on the mesentery usually within $2 \mathrm{~mm}$ of the colon. ${ }^{2}$ Serosal afferents responded to blunt probing but not to mucosal stroking or circular muscle stretch, except for a few action potentials at high intensity stretch. ${ }^{2}$ Mesenteric afferents responded in a graded manner to blunt probing with calibrated von Frey hairs. Stimulus-response functions were constructed by assessing the average number of action potentials/second during the response to probing with calibrated von Frey hairs $(70,160,1000,2000$, and $4000 \mathrm{mg}$ ) for 3 seconds each. Mean frequency and instantaneous frequency were also measured and compared. Adaptation during the probing response was assessed by dividing it into $200 \mathrm{~ms}$ bins and expressing it as a percentage of the maximum response. Responses to low $\mathrm{pH}$ were not evaluated in colonic or gastro-oesophageal afferents because of their infrequent incidence and variable intensity, ${ }^{18}$ and the focus of this investigation on mechanosensory function.

\section{In vitro gastro-oesophageal vagal afferent nerve recording}

Gastro-oesophageal mechanoreceptors were recorded using a single fibre technique similar to that for colonic afferents, which has been described in detail previously. ${ }^{1}$ Briefly, receptive fields were identified by systematically stroking the mucosal surface of the oesophagus and stomach with a fine brush. They were then classified into mucosal and tension receptors according to their responses to calibrated von Frey hairs (10-1000 mg) stroked over the mucosa and circumferential stretch applied across the receptive field using a hook and pulley/cantilever system (0.5-5 g; fig 2).

\section{Studies of digestive function in conscious mice \\ Non-invasive breath test of gastric emptying}

Gastric emptying time of a solid meal was determined using non-invasive breath tests as previously described. ${ }^{19}{ }^{20}$ Briefly, after an overnight fast and following baseline breath sample collection, ASICla, 2, and $3+/+$ and -/- mice voluntarily consumed a nutrient solid meal consisting of $0.1 \mathrm{~g}$ of baked egg yolk containing $1 \mu \mathrm{l} / \mathrm{g}{ }^{13} \mathrm{C}$-octanoic acid $(99 \%$
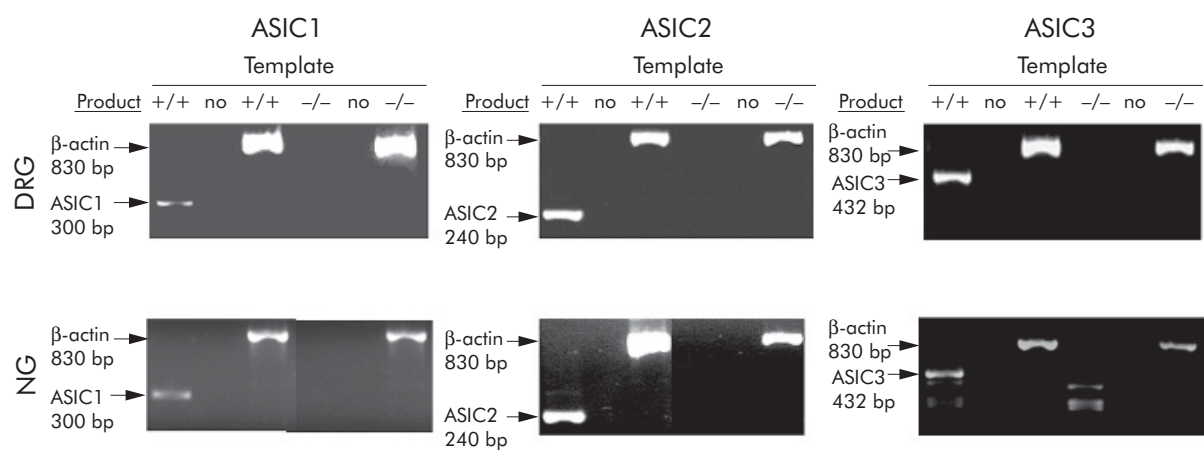

$\beta$-actin $830 \mathrm{bp} \rightarrow$ ASIC3$432 \mathrm{bp}$

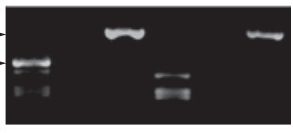

Figure 1 PCR of sensory ganglia in ASIC wild-type and null mutant mice. ASIC 1a, 2, and 3 were all detected in lumbar dorsal root ganglia (DRG) and nodose ganglia (NG) of wild-type mice $(+/+)$, but were not detectable in null mutants of each gene (-/-). Where the RNA template was substituted with distilled water (no), no evidence of amplified product was seen in wildtypes or null mutants, and expression of the housekeeping gene $\beta$-actin was observed in all cases. Note that ASIClb expression persists in the $\mathrm{ASICla}$ knockout, ${ }^{10}$ but was not detectable with the PCR conditions and primers used in this study. 
A Gastro-oesophageal
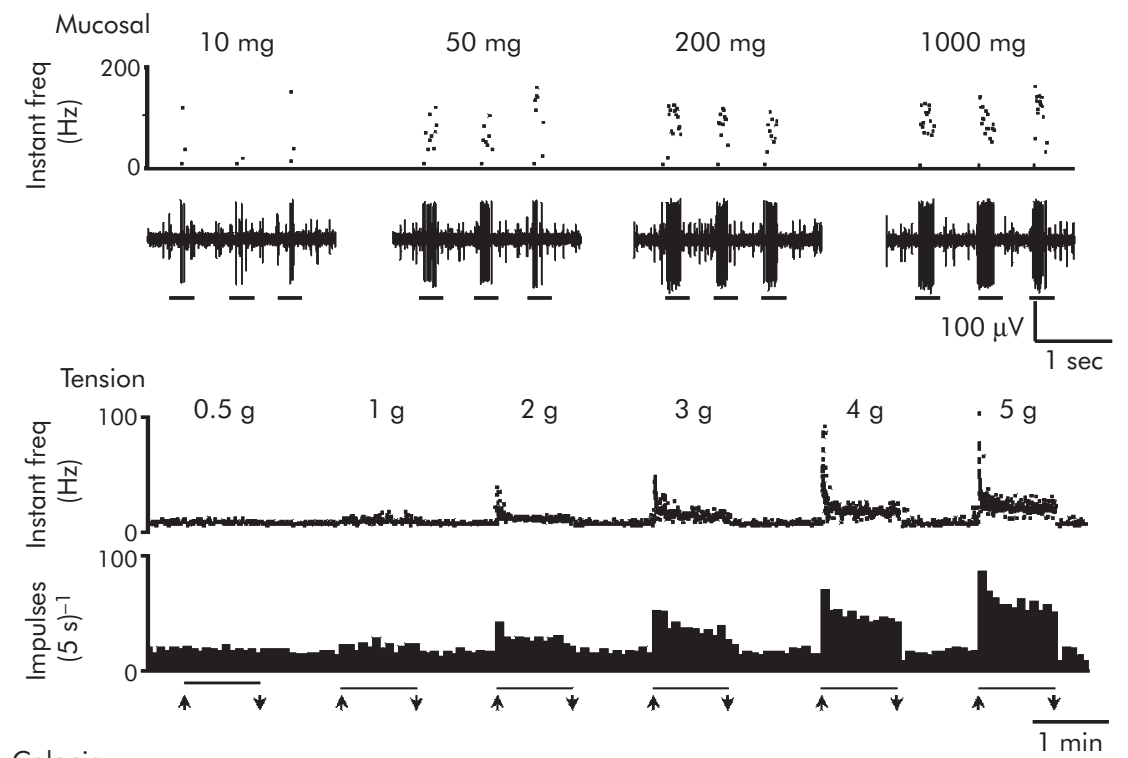

B Colonic
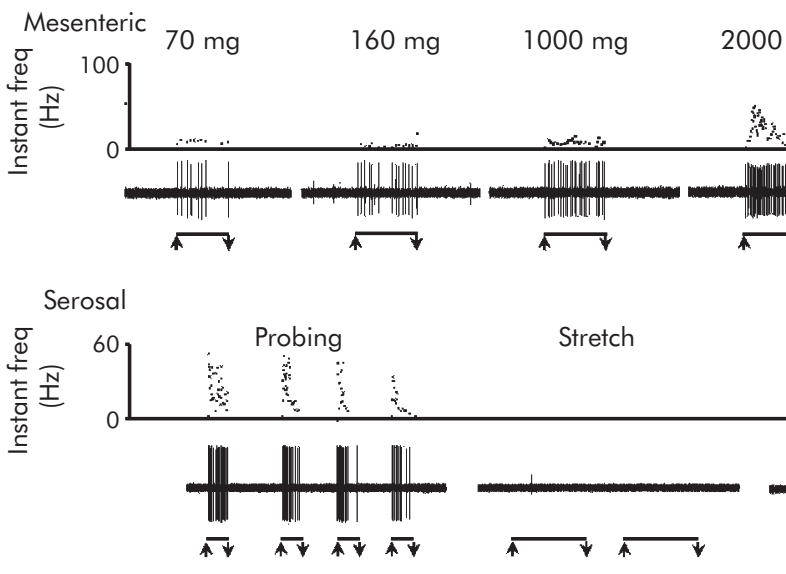

Figure 2 Representative traces of wild-type visceral afferent mechanosensitivity. (A) Gastrooesophageal afferents: mucosal receptors and tension receptors. Mucosal receptors showed mechanical thresholds below $10 \mathrm{mg}$, and gave graded responses to von Frey hairs up to $1000 \mathrm{mg}$ applied by stroking the mucosa during the periods indicated by bars. Tension receptors showed slowly adapting responses to maintained force across the receptive field applied via a claw attached to the edge of the sheet preparation, during the periods indicated by bars; they had thresholds of $<0.5 \mathrm{~g}$ tension and gave maximal responses at $4-5 \mathrm{~g}$ tension. (B) Colonic afferents: mesenteric and serosal mechanoreceptors. Mesenteric mechanoreceptors were located on the colonic mesenteric attachment and responded in a graded manner to an ascending series of perpendicular probing stimuli $(70 \mathrm{mg}-4 \mathrm{~g}$ von Frey hairs). Serosal mechanoreceptors were located within the colonic wall and were activated only by perpendicular probing of their receptive fields (with a $2 \mathrm{~g}$ von Frey hair in this example) and were insensitive to circular stretch $(5 \mathrm{~g})$ and fine mucosal stroking $(10 \mathrm{mg})$, all indicated by bars. Upper traces show instantaneous firing frequency and lower traces show raw electrophysiological recordings or integrated responses as spikes/bin. enrichment, Cambridge Isotope Laboratories, Andover, MA, USA). Only mice that consumed the meal at the first attempt were included, and no differences in behaviour during consumption were noted. Breath samples were collected at intervals for a total of 150 minutes, and analysed for ${ }^{13} \mathrm{CO}_{2}$ content with an isotope ratio mass spectrometer (Europa Scientific, Crewe, UK). The ${ }^{13} \mathrm{CO}_{2}$ excretion data were analysed by non-linear regression analysis for curve fitting and for calculation of gastric half emptying time $\left(t_{1 / 2}\right){ }^{21}$

Faecal pellet output

Pellet output was monitored in four mice of each genotype housed in wire bottomed cages, and results averaged over 34 days observation. Number of pellets per day and total pellet weight were measured and individual pellet weight was calculated. Pellet form was qualitatively observed for changes in consistency or shape.

\section{Data analysis}

Differences between stimulus response functions and adaptation curves were assessed using two way analysis of variance (ANOVA), with repeated measures Bonferroni post hoc tests for differences at individual stimulus strengths and time points respectively. In addition, linear regression was performed on adaptation curves. Gastric emptying rates, faecal output, and spontaneous discharge of afferents were compared by unpaired $t$ test. Data are expressed as mean (standard error of mean). $\mathrm{N}=$ number of animals and $\mathrm{n}=$ number of observations.

\section{RESULTS}

ASIC expression in vagal and spinal sensory ganglia RT-PCR analysis of spinal (dorsal root) and vagal (nodose) ganglia, which innervate the colon and stomach respectively, indicated expression of all three ASIC subtypes in both locations. Disruption of ASIC1, 2, or 3 genes resulted in loss of the corresponding transcript in all cases (fig 1).

\section{Effects of ASIC disruption on visceral mechanoreceptors}

The responses of wild-type gastro-oesophageal and colonic afferents are illustrated in figure 2 . In the vagal innervation of the mouse stomach and oesophagus we found mucosal and tension receptors. Mucosal receptors were silent in the absence of stimulation, and responded exclusively to fine tactile stimuli ( $10 \mathrm{mg}$ von Frey hair) with rapidly adapting responses (fig $2 \mathrm{~A}$ (top)). Tension receptors were spontaneously active, and responded to muscle stretch with slowly 
Gastro-oesophageal afferents: ASIC1
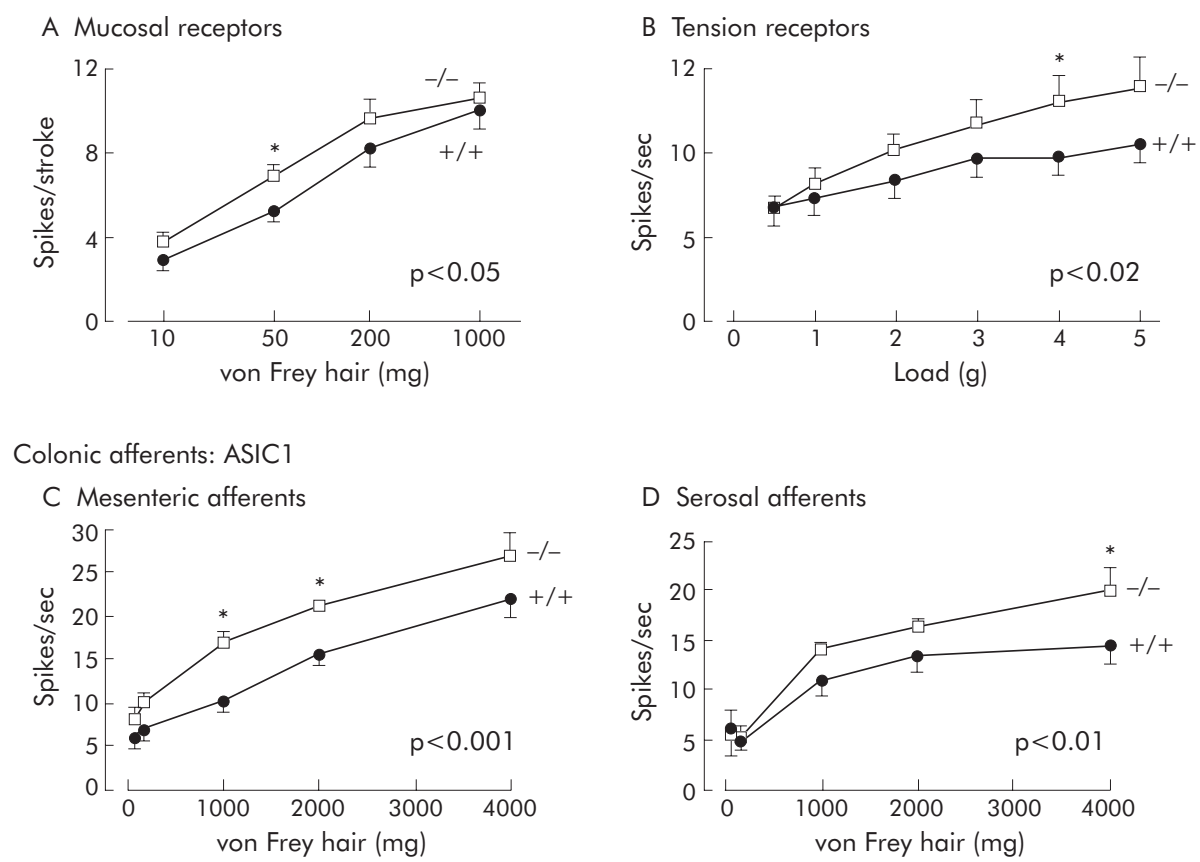

Figure 3 Effects of disrupting ASICla on visceral mechanoreceptors. (A) Increased sensitivity of vagal gastro-oesophageal mucosal receptors. Stimulus-response functions of mucosal receptors from ASICla (+/+) (circles, $n=18, N=12)$ and $(-/-)$ mice (squares, $n=28, N=13)$, showing a significant increase in ASICla -/- (p<0.05, two way ANOVA of stimulus response curves). (B) Increased sensitivity of vagal gastro-oesophageal tension receptors. Stimulus-response functions of tension receptors from $\mathrm{ASICla}(+/+)$ (circles, $n=40, N=23)$ and $(-/-)$ mice $($ squares, $n=47, N=30)$, showing a significant increase in ASICla -/- $\left(p<0.02\right.$, two way ANOVA; data from Page et a $\left.{ }^{\circ}\right)$. (C) Increased sensitivity of colonic mesenteric mechanoreceptors. Stimulus-response functions of colonic mesenteric mechanoreceptors from ASICla $(+/+)$ (circles, $n=10, N=8)$ and $(-/-)$ mice (squares, $n=17, N=11$ ), showing a significant increase in stimulus-response function in ASICla -/- mice $(p<0.001$, two way ANOVA). (D) Increased sensitivity of colonic serosal mechanoreceptors and unaffected adaptation profile. Stimulus-response functions of colonic serosal mechanoreceptors from ASICla $(+/+)$ (circles, $n=17, N=12)$ and $(-/-)$ mice (squares, $n=16, N=11)$, showing a significant increase in stimulus-response function in $\mathrm{ASICla}-/-$ mice $(\mathrm{p}<0.01$, two way ANOVA). Asterisks denote differences at individual stimulus strengths significant by Bonferroni post hoc analysis $\left({ }^{*} \mathrm{p}<0.05\right)$. $\mathrm{N}=$ number of animals and $\mathrm{n}=$ number of observations.

adapting responses (fig 2A (bottom)). In both cases responses were graded according to the intensity of the mechanical stimulus (see also figs $3 \mathrm{~A}$ and $\mathrm{B}, 4 \mathrm{~A}$ and $\mathrm{B}, 5 \mathrm{~A}$ and $\mathrm{B}$, and Page et $\mathrm{al}^{1}$ ).

In the splanchnic innervation of the mouse colon, we found four classes of afferents: mucosal, muscular, serosal, and mesenteric. The latter two comprised the large majority of the population and were selected for studies of gene disruption. Serosal and mesenteric afferents responded to probing of the serosa or mesentery (fig 2B) with graded responses (fig 2B (top), 3C and D, 4C and D, 5C and D); see also Brierley et al. ${ }^{2}$ Both classes displayed little or no spontaneous activity and neither responded to mucosal stroking nor to muscle stretch with maintained responses (for example, fig 2B (bottom)).

\section{Effects of ASIC1 disruption}

Compiled data for effects of ASICI gene disruption on mechanical stimulus response functions and on adaptation are shown in figure 3 and summarised in table 2. As we have shown previously, ${ }^{10}$ both gastro-oesophageal mucosal and tension receptors displayed increased sensitivity as indicated by their increased stimulus response functions. Parameters that were unaltered in ASICla-/- mice were adaptation (not shown), spontaneous discharge of tension receptors (ASIClat/+ 4.0 (SEM 0.6) $v$ - $/$ - 3.3 (SEM 0.4) impulses/ second, $\mathrm{p}>0.05$ by unpaired $t$ test), and mechanical thresholds (not shown).

Similarly ASICl gene disruption increased sensitivity in colonic mesenteric and serosal afferents, as indicated by increased stimulus response functions (fig $3 \mathrm{C}$ and D). This was significant by two way ANOVA $(p<0.01$ to 0.001$)$ of stimulus-response curves. Post hoc analysis showed that only higher stimulus strengths were associated with significant differences in response (fig 3C and D). A small but significant alteration in the adaptation profile of mesenteric afferents was evident ( $\mathrm{p}<0.05$, linear regression; not illustrated), but no significant change occurred in that of serosal afferents. This became evident after performing studies additional to those we have previously published on ASICla null mutants, ${ }^{10}$ which enabled us to characterise separately the two subtypes of colonic afferents as mesenteric and serosal afferents. The importance of separating these subtypes became apparent when other gene disruptions were investigated (see later). As with gastro-oesophageal afferents, no changes in threshold or spontaneous discharge were observed (data not shown).

\section{Effects of ASIC2 disruption}

ASIC2 compiled data are shown in figure 4 and summarised in table 2. A similar increase in the stimulus response functions of gastro-oesophageal mucosal receptor sensitivity was seen to that observed in ASICla null mutants (compare figs $3 \mathrm{~A}$ and $4 \mathrm{~A}$ ). Tension receptors on the other hand showed a marked decrease in stimulus response function by approximately half. Neither their adaptation rate (not shown), their spontaneous discharge (ASIC2+/+ 6.28 (SEM 1.94) $v$-/- 4.47 (SEM 0.55) impulses/second), nor their thresholds (not shown) were significantly affected $(p>0.05)$.

Colonic afferents were affected differently by ASIC2 disruption according to the subclass of fibres. Mesenteric afferents were unaffected (fig 4C), whereas serosal afferents 
Gastro-oesophageal afferents: ASIC2

A Mucosal receptors

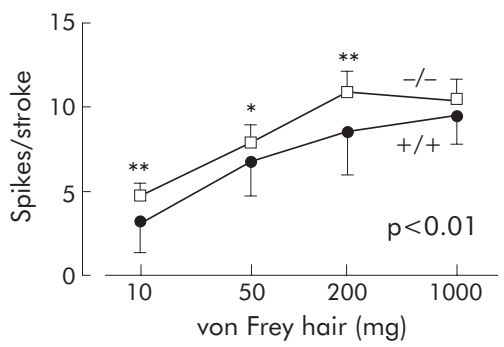

Colonic afferents: ASIC2

C Mesenteric afferents

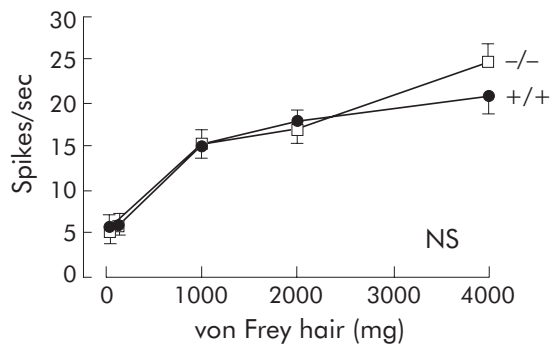

B Tension receptors

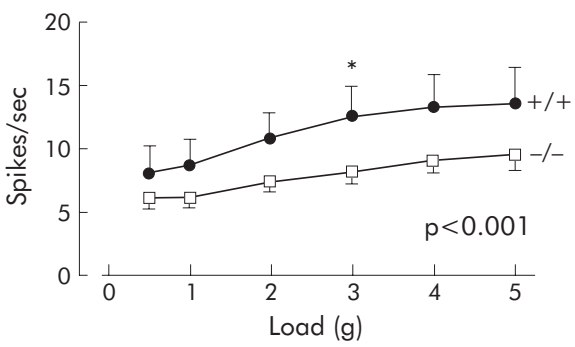

D Serosal afferents

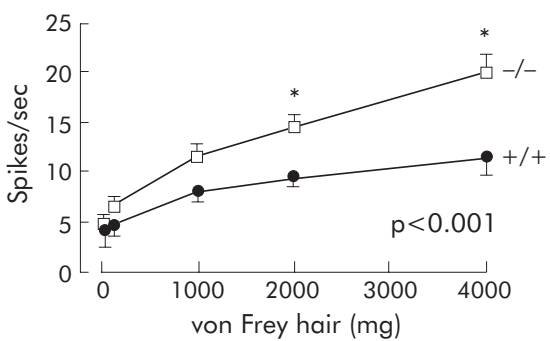

Figure 4 Effects of disrupting ASIC2 on visceral mechanoreceptors. (A) Increased sensitivity of vagal gastro-oesophageal mucosal receptors. Stimulus-response functions of mucosal receptors from ASIC2 $(+/+)$ (circles, $n=23, N=8)$ and $(-/-)$ mice (squares, $n=21, N=12)$, showing a significant increase in ASIC2 -/- ( $<0.01$, two way ANOVA of stimulus response curves). (B) Decreased sensitivity of vagal gastro-oesophageal tension receptors. Stimulus-response functions of tension receptors from ASIC2 (+/+) (circles, $n=23, N=8)$ and $(-/-)$ mice $($ squares, $n=21, N=12)$, showing a significant decrease in ASIC2 -/- ( $<<0.001$, two way ANOVA). (C) Unaltered sensitivity of colonic mesenteric mechanoreceptors. Stimulusresponse functions from ASIC2 (+/+) (circles, $n=20, N=14$ ) and (-/-) mice (squares, $n=26, N=15)$, showing no change in stimulus-response function ( $>0.05$, two way ANOVA). (D) Increased sensitivity of colonic serosal mechanoreceptors. Stimulus-response functions of colonic serosal mechanoreceptors from ASIC2 (+/+) (circles, $\mathrm{n}=27, \mathrm{~N}=19)$ and $(-/-)$ mice (squares, $\mathrm{n}=34, \mathrm{~N}=21$ ), showing a significant increase in stimulusresponse function in ASIC2 -/- mice $(\mathrm{p}<0.01$, two way ANOVA). Asterisks denote differences at individual stimulus strengths significant by Bonferroni post hoc analysis ( $\left.{ }^{*} \mathrm{p}<0.05,{ }^{* *} \mathrm{p}<0.01\right)$. $\mathrm{N}=$ number of animals and $\mathrm{n}=$ number of observations.

became more sensitive, with almost double the response to mechanical stimulation (fig 4D). Other parameters (adaptation, threshold, and spontaneous activity) were not affected for either class of afferent.

\section{Effects of ASIC3 disruption}

Figure 5 shows the responses of gastro-oesophageal and colonic afferents in ASIC3 +/+ and -/- mice. Gastrooesophageal mucosal afferents showed no significant differences between genotypes (fig 5A, table 2), whereas tension receptors were consistently reduced in sensitivity, without alteration in their rate of adaptation (not shown), or spontaneous activity (ASIC3+/+ 4.55 (SEM 0.68) $v$-/- 3.27 (SEM 0.48) impulses/second, not significant).

Colonic afferents were affected in a similar way to gastrooesophageal tension receptors, with both showing considerably reduced sensitivity (fig 5C and D), and mesenteric afferents showing slightly faster adaptation over three seconds $(\mathrm{p}<0.05$, linear regression, not shown).

\section{Effects of ASIC disruption on digestive function}

Effects on gastric emptying and faecal pellet output Of all the three null mutants we studied, only ASICla showed alteration in upper gastrointestinal function compared with wild-types. This was manifested as a twofold increase in gastric emptying $\mathrm{t} 1 / 2$, (fig 6; data from Page et $a l^{10}$ ). On the other hand, changes in lower bowel function were only evident in ASIC2 null mutants, with a significant decrease in the number of faecal pellets/day (fig 6B). This tended to be accompanied by a slight increase in the weight of each pellet, although the difference was not statistically significant (not shown). Thus, the weight of total faecal output was normal in these animals, and none of them showed signs of chronic retention of material in any part of the gut, suggesting that they had adapted to changes in gastric emptying or pellet formation.

\section{DISCUSSION \\ Contribution of ASICs to visceral mechanotransduction}

This study demonstrates four major distinct subtypes of mechanoreceptors innervating the upper and lower gastrointestinal tract of mice, confirming our previous findings. ${ }^{12}$ Widespread changes in sensitivity of these mechanoreceptors occurred with disruption of ASICla, 2, or 3, indicating that they are all required for normal mechanotransduction. The contribution of ASICs to mechanotransduction is most likely reflected in events that are linked with mechanoreceptor activation, such as perception, behaviour, and ultimately in reflex responses of the gut. In this respect we found in some cases that disrupting ASIC genes altered digestive activity in the whole animal by disrupting emptying patterns from the upper or lower gastrointestinal tract. Our findings of both positive and negative effects of ASIC mutations on mechanosensitivity suggest a complexity in the way they contribute to mechanotransduction. It is therefore unlikely that ASICs function simply as individual mechanically gated cation channels, and in some cases they may in fact dampen the mechanotransduction process. This would be the case for ASIC2 in colonic afferents, and for ASICla in all the populations we studied, because in both instances mechanosensitivity was increased in null mutants. This negative modulatory or "dampening" role is probably a result of existence of all three ASIC subtypes as heteromultimers, in 
Gastro-oesophageal afferents: ASIC3
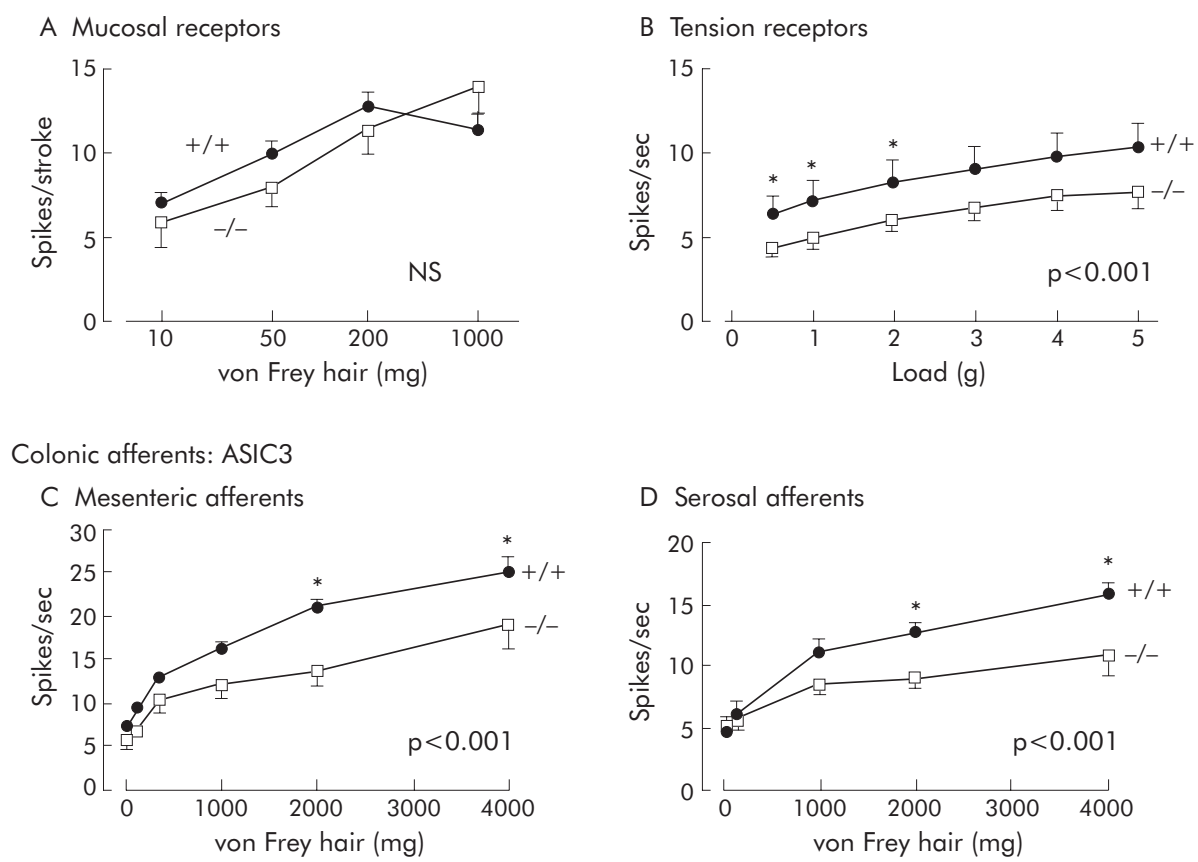

Figure 5 Effects of disrupting ASIC3 on visceral mechanoreceptors. (A) Unaltered sensitivity of vagal gastro-oesophageal mucosal receptors. Stimulus-response functions of mucosal receptors from ASIC3 (+/+) (circles, $n=26, N=12$ ) and (-/-) mice (squares, $n=12, N=9)(N S=p>0.05$, two way ANOVA). (B) Decreased sensitivity of vagal gastro-oesophageal tension receptors. Stimulus-response functions of tension receptors from ASIC3 $(+/+)$ (circles, $n=37, N=8)$ and $(-/-)$ mice (squares, $n=37, N=8)$, showing a significant decrease in ASIC3 -/- $(p<0.001$, two way ANOVA of stimulus response curves). (C) Decreased sensitivity of colonic mesenteric mechanoreceptors. Stimulus-response functions of colonic mesenteric mechanoreceptors from ASIC3 $(+/+)$ (circles, $n=23, N=14)$ and $(-/-)$ mice (squares, $n=26, N=15)$, showing a significant decrease in stimulusresponse function in ASIC3 -/- mice ( $p<0.001$, two way ANOVA). (D) Decreased sensitivity of colonic serosal mechanoreceptors. Stimulus-response functions of colonic serosal mechanoreceptors from ASIC3 (+/+) (circles, $n=26, N=15)$ and $(-/-)$ mice (squares, $n=23, N=14$ ) showing a significant decrease in stimulus-response function in ASIC3 -/- mice ( $p<0.001$, two way ANOVA). Asterisks denote differences at individual stimulus strengths significant by Bonferroni post-hoc analysis ( $\left.{ }^{*} p<0.05\right)$. Note that although mechanosensitivity was markedly reduced in $B, C$, and D in ASIC3 -/- mice, stimulus-response functions were significant by repeated measures one way ANOVA ( $<<0.05$ to $p<0.001$ ), indicating graded responses to mechanical stimuli were evoked in these mice despite the shallower curves. $N=$ number of animals and $n=$ number of observations.

which each member contributes directly or indirectly to mechanotransduction. ASICla clearly appears to make little if any direct contribution to mechanotransduction, because without it mechanosensitivity is universally increased in visceral afferents, suggesting that the heteromultimeric mechanotransducer becomes more efficient. ASIC3 appears to make a positive contribution in most cases, whereas the role of ASIC2 is intriguing in its capacity to influence mechanosensitivity negatively in lower gut afferents and positively in upper gut afferents. More evidence for ASICs forming functional heteromultimers arises from studies of recombinant and native channels. ${ }^{3}{ }^{16}$ These showed that low $\mathrm{pH}$-induced currents in cells expressing ASICl, 2, or 3 individually showed quite different characteristics from the current in native sensory neurones, even if the three currents were mathematically summed. On the other hand, the low pH-induced current in cells coexpressing all three ASICs was identical to the native response, indicating some interaction between them must occur. In studies such as the present using gene disruption, interpretation is complicated because when one of the channels is lost from the heteromultimeric complex, the others may compensate either by changes in expression levels or by reassembly into different configurations. We investigated the up- or downregulation of other subunits in previous quantitative studies of ASIC expression, and showed that ASIC 1 and 2 levels are unchanged in ASIC3 null mutants, and ASIC 1 and 3 levels are unchanged in ASIC2 null mutants. ${ }^{11}{ }^{12}$ The possibility remains that there may be changes in subunit configuration other than just the subtraction of the disrupted channel. Pharmacological studies may ultimately resolve this issue if highly selective blockers for individual ASIC subtypes become available, which will allow investigation of their contribution in intact heteromultimers. Some candidates are already emergingrecently an ASIC3 selective compound was discovered in sea anemone. ${ }^{22}$

Table 2 Summary of effects of ASIC disruption on mechanosensory responses in each afferent subtype and effects on digestive function in conscious animals

\begin{tabular}{lllllll}
\hline & \multicolumn{2}{l}{ Gastro-oesophageal } & & Gastric & & \multicolumn{2}{c}{ Colonic } & & Faecal pellet \\
\cline { 2 - 3 } & Mucosal & Tension & emptying & Serosal & Mesenteric & output \\
\hline ASICla & $\uparrow$ & $\uparrow$ & $\downarrow$ & $\uparrow$ & $\uparrow$ & $\leftrightarrow$ \\
ASIC2 & $\uparrow$ & $\downarrow \downarrow$ & $\leftrightarrow$ & $\uparrow \uparrow$ & $\leftrightarrow$ & $\downarrow$ \\
ASIC3 & $\leftrightarrow$ & $\downarrow \downarrow$ & $\leftrightarrow$ & $\downarrow \downarrow$ & $\downarrow \downarrow$ & $\leftrightarrow$ \\
\hline
\end{tabular}


A ASICl Solid gastric emptying
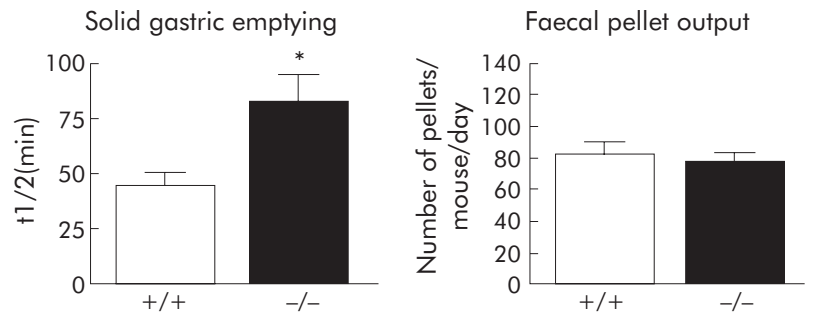

B ASIC2

Solid gastric emptying
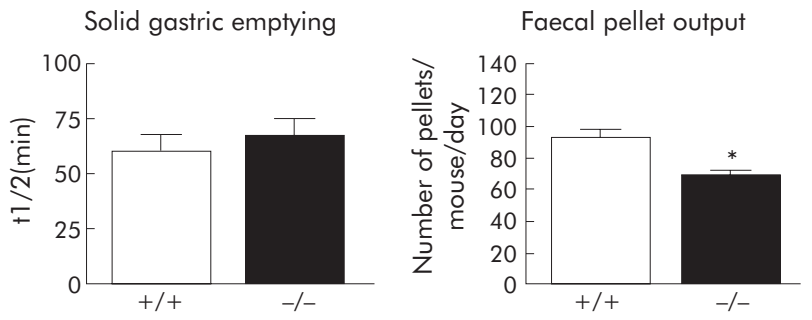

C ASIC3

Solid gastric emptying
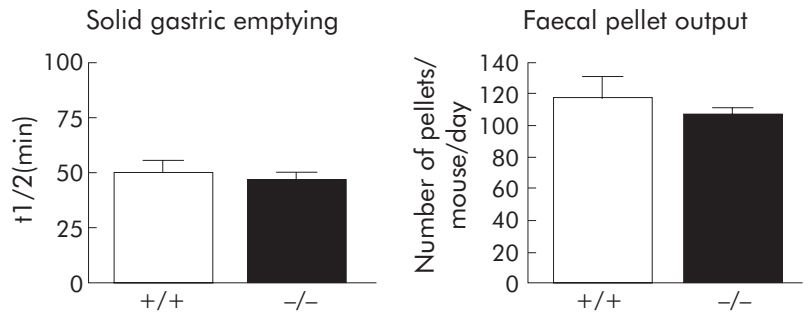

Figure 6 Effects of disrupting ASIC 1, 2, or 3 on markers of digestive function in conscious mice. (A) Left: increased time for half gastric emptying $(t 1 / 2)$, indicating a delay in $\mathrm{ASICla}-/$ - mice compared with wild-types $(p<0.05$, unpaired t test; $(+/+) N=8,(-/-) N=14)$. These data are taken from Page et al. ${ }^{10}$ Right: unaltered faecal pellet output in $\mathrm{ASICla}$-/- mice compared to wild-types ( $N=3$ each). (B) Left: unaltered gastric emptying $\left(t^{1} / 2\right)$ in ASIC2 - /- mice compared with wild-types (NS; $\mathrm{N}=10$ each). Right: decreased faecal pellet output in ASIC2 -/- mice compared with wild-types ( $p<0.05$, unpaired $t$ test; $N=5$ each). (C) Left: unaltered gastric emptying $(t 1 / 2)$ in ASIC3 -/- mice compared with wildtypes (NS; $(+/+) N=11,(-/-) N=10)$. Right: unaltered faecal pellet output in ASIC3 -/- mice compared with wild-types ( $\mathrm{N}=5$ each).

A comparison of results from the present study with those of previous investigations reveals contrasting contributions of ASICs in visceral and cutaneous mechanotransduction. Such a comparison is possible because we have consistently used a direct approach of measuring mechanosensitivity in single primary afferent fibres with identified receptive fields in the gut or skin, ${ }^{10-12}$ and correlating this with patterns of digestive and behavioural function in the conscious animal. Our evidence indicates that ASICla makes a consistent negative contribution in all classes of visceral afferents, whereas no contribution was evident in any of the five classes of cutaneous afferents. ${ }^{10}$ Disruption of ASIC2 reduced mechanosensitivity in two out of five cutaneous mechanoreceptor types, and had no effect on the other three. ${ }^{11}$ In viscera, ASIC2 disruption led to potent increases in sensitivity of colonic splanchnic serosal afferents and potent decreases in gastrooesophageal vagal tension receptors. Mesenteric afferents in colon and mucosal afferents in upper gut were affected slightly if at all. Therefore the contribution of ASIC2 is specific to individual fibre types and regions of gut, and is notably opposite in colon and skin. Another study on the role of ASIC2 in viscera used colonic stretch-induced peptide release as an indirect measure of mechanosensation, ${ }^{23}$ and found no change in ASIC2 null mutants. This suggests that stretch sensitive colonic afferents may be similar to mesenteric colonic afferents in their lack of requirement for ASIC2. The predominant effect of ASIC3 disruption in skin was an increased sensitivity of RA mechanoreceptors. ${ }^{12}$ In contrast, it potently decreased mechanosensitivity in three out of four classes of visceral afferents, indicating a widespread positive influence. This suggests ASIC3 is an important potential target for modulating mechanosensory function in the gut, particularly in colonic high threshold afferents (see fig 5). Our data indicate that there is a high degree of tissue and pathway specificity to the contributions of ASICs to mechanotransduction. It is worthy of note that fibres respond in a graded manner regardless of pathway or genotype. However, responsiveness of colonic afferents to weak stimuli is relatively unchanged in knockouts yet their responses to higher stimulus intensities are markedly altered, suggesting a greater contribution of ASICs as noxious intensities are reached. A study on cell bodies of DRG neurones indicated no change in their responses to probing in ASIC2 and 3 single and double knockouts. ${ }^{24}$ Combined with our current and previous findings, ${ }^{11}{ }^{12}$ these data indicate that the mechanisms of activation of primary afferents may be different at their peripheral endings and in their cell bodies. Despite the weight of evidence from mammalian and non-mammalian research in favour of ASICs as molecular mechanotransducers at sensory endings, it is conceivable that they may be involved indirectly by modulating the activity of other mechanisms. Whether their role is direct or indirect, their clinical and biological significance to the process of visceral mechanotransduction is becoming clear.

\section{Clinical and biological significance of ASICs}

A number of diseases involving the gastrointestinal system may benefit from altered signaling from vagal or spinal mechanoreceptors. In obesity, various strategies are currently used to maximise stimulation of gastric vagal mechanoreceptors, such as surgical reduction of the distensible volume of the stomach, which lead to increased satiety signals and reduced food intake. ${ }^{25}$ In gastro-oesophageal reflux disease, reduced signaling from proximal gastric mechanoreceptors leads to reduced triggering of the sphincter relaxations underlying reflux events. ${ }^{26}$ In irritable bowel syndrome, one of the hallmarks of disease is an increased perception of phasic distension of the colon and rectum, ${ }^{27} 28$ so it too would benefit from a reduction in mechanoreceptor signaling. A number of clinical situations involving bowel obstruction or blockage of ducts may lead to intense pain. ${ }^{29}$ Therefore either positive or negative modulation of the mechanotransduction process in visceral mechanoreceptors is a highly desirable option, depending on the clinical problem.

The gut optimises its motor activity to enable efficient transit of contents from one region to the next. The stomach and colon are the regions most specialised for storage of content and they are controlled by continual feedback from neural reflexes. Disturbed gastric coordination and delayed emptying are often seen following stimulation of gastric and intestinal mechanoreceptors, ${ }^{30}$ and colonic motility may be reflexly inhibited by colonic mechanical stimuli. ${ }^{31}$ When we increased the sensitivity of gut mechanoreceptors by disruption of ASICl and 2, there were consequences for digestive function in the whole animal. Thus, increased gastrooesophageal mechanoreceptor function in ASICla null mutants correlates with decreased gastric emptying. Increased colonic afferent sensitivity in ASIC2 null mutants was associated with decreased numbers of faecal pellets, presumably due to altered feedback control of colonic motility. The observation that reduced faecal output occurred in ASIC2-/- mice but not in ASICla-/-, which also had increased colonic afferent sensitivity, may relate to the 
importance of specific patterns of increases and decreases in mechanoreceptor function across the different populations of afferents. Although we observed reduced distal progression of digesta from storage areas (stomach or colon), there was no long term retention of gut content, indicating that the behaviour of the gut overall was able to compensate for these delays. In this respect the role of ASICs in mechanosensory function in intrinsic neurones of the gut remains to be investigated. The decrease in gastro-oesophageal and colonic mechanoreceptor sensitivity in ASIC3 null mutants did not translate to changes in gastric emptying or faecal pellet output. This is consistent with the role of mechanoreceptor feedback in gut reflexes mainly as a braking mechanism to delay onward propulsion of digesta so that more distal segments can be prepared for its arrival, optimising digestive capacity.

In conclusion, disruption of ion channels of the ASIC family has varied but consistent effects on mechanosensitivity in a range of visceral mechanoreceptors. From this and previous studies we have a comprehensive picture of the effects of $A S I C 1,2$, and 3 disruption on the function of visceral and cutaneous sensory fibres, and can conclude that the consequences are, in general, greater for visceral mechanoreceptor function than for cutaneous mechanoreceptor function. The different roles of ASIC subtypes in particular classes of visceral mechanoreceptors may be important in targeting pharmacotherapy towards increasing or decreasing afferent signals from specific regions of gut.

\section{ACKNOWLEDGEMENTS}

Supported by the National Health and Medical Research Council of Australia and the Howard Hughes Medical Institute (HHMI). Professor Michael Welsh is gratefully acknowledged for helpful discussion.

\section{Authors' affiliations}

A J Page, S M Brierley, C M Martin, L A Blackshaw, Nerve-Gut Research Laboratory, Hanson Institute, Department of Gastroenterology, Hepatology and General Medicine, Royal Adelaide Hospital, Adelaide, SA, Australia

A J Page, S M Brierley, C M Martin, R Butler, L A Blackshaw, Discipline of Physiology, University of Adelaide, Adelaide, SA, Australia A J Page, L A Blackshaw, Department of Medicine, University of Adelaide, Adelaide, SA, Australia

J A Wemmie, Department of Psychiatry, Department of Veterans Affairs Medical Centre

M P Price, Department of Physiology \& Biophysics and Howard Hughes Institute, University of lowa College of Medicine, lowa City, lowa, USA E Symonds, R Butler, Centre for Paediatric and Adolescent Gastroenterology, Women's and Children's Hospital, North Adelaide, SA, Australia

Competing interests: no competing interests are declared.

Ethics approval: All procedures were approved by the Animal Ethics Committees of the Institute of Medical and Veterinary Science, Adelaide and the University of Adelaide.

*These authors contributed equally to this work

\section{REFERENCES}

1 Page AJ, Martin CM, Blackshaw LA. Vagal mechanoreceptors and chemoreceptors in mouse stomach and esophagus. J Neurophysiol 2002;87:2095-103.

2 Brierley SM, Jones RC 3rd, Gebhart GF, et al. planchnic and pelvic mechanosensory afferents signal different qualities of colonic stimuli in mice. Gastroenterology 2004;127:166-78.
3 Welsh MJ, Price MP, Xie J. Biochemical basis of touch perception: mechanosensory function of degenerin/epithelial $\mathrm{Na}+$ channels. J Biol Chem 2002;277:2369-72.

4 Gillespie PG, Walker RG. Molecular basis of mechanosensory transduction. Nature 2001;413:194-202.

5 Rong W, Hillsley K, Davis JB, et al. Jejunal afferent nerve sensitivity in wildtype and TRPV1 knockout mice. J Physiol 2004;560:867-81.

6 Suzuki M, Mizuno A, Kodaira K, et al. Impaired pressure sensation in mice lacking TRPV4. J Biol Chem 2003;278:22664-8.

7 Corey DP, Garcia-Anoveros J, Holt JR, et al. TRPA1 is a candidate for the mechanosensitive transduction channel of vertebrate hair cells. Nature 2004;432:723-30.

8 Chen CC, England S, Akopian AN, et al. A sensory neuron-specific, protongated ion channel. Proc Nat Acad Sci U S A 1998;95:10240-5.

9 Garcia-Anoveros J, Samad TA, Zuvela-Jelaska L, et al. Transport and localization of the $\mathrm{DEG} / \mathrm{ENaC}$ ion channel $\mathrm{BNaCl}$ alpha to peripheral mechanosensory terminals of dorsal root ganglia neurons. J Neurosci 2001;21:2678-86.

10 Page AJ, Brierley SM, Martin CM, et al. The ion channel ASICl contributes to visceral but not cutaneous mechanoreceptor function. Gastroenterology 2004; 127:1739-47.

11 Price MP, Lewin GR, Mcllwrath SL, et al. The mammalian sodium channel BNC1 is required for normal touch sensation. Nature 2000;407:1007-11.

12 Price MP, Mcllwrath SL, Xie J, et al. The DRASIC cation channel contributes to the detection of cutaneous touch and acid stimuli in mice. Neuron 2001;32:1071-83.

13 Waldmann R, Bassilana F, de Weille J, et al. Molecular cloning of a noninactivating proton-gated $\mathrm{Na}+$ channel specific for sensory neurons. J Biol Chem 1997;272:20975-8.

14 Alvarez de la Rosa D, Zhang P, Shao D, et al. Functional implications of the localization and activity of acid-sensitive channels in rat peripheral nervous system. Proc Natl Acad Sci U S A 2002;99:2326-31

15 Driscoll M, Chalfie M. The mec- 4 gene is a member of a family of Caenorhabditis elegans genes that can mutate to induce neuronal degeneration. Nature 1991;349:588-93.

16 Benson CJ, Xie J, Wemmie JA, et al. Heteromultimers of DEG/ENaC subunits form $\mathrm{H}+$-gated channels in mouse sensory neurons. Proc Natl Acad Sci U S A 2002;99:2338-43.

17 Wemmie JA, Chen J, Askwith CC, et al. The acid-activated ion channel ASIC contributes to synaptic plasticity, learning, and memory. Neuron 2002;34:463-77

18 Lynn PA, Blackshaw LA. In vitro recordings of afferent fibres with receptive fields in the serosa, muscle and mucosa of rat colon. J Physiol 1999;518:271-82

19 Symonds EL, Butler RN, Omari TI. Assessment of gastric emptying in the mouse using the [13C]-octanoic acid breath test. Clin Exp Pharmacol Physiol 2000;27:671-5

20 Symonds E, Butler R, Omari T. Noninvasive breath tests can detect alterations in gastric emptying in the mouse. Eur J Clin Invest 2002;32:341-4.

21 Ghoos YF, Maes BD, Geypens BJ, et al. Measurement of gastric emptying rate of solids by means of a carbon-labeled octanoic acid breath test. Gastroenterology 1993;104:1640-7.

22 Diochot S, Baron A, Rash LD, et al. A new sea anemone peptide, APETx2, inhibits ASIC3, a major acid-sensitive channel in sensory neurons. Embo $J$ 2004;23:1516-25.

23 Roza C, Puel JL, Kress M, et al. Knockout of the ASIC2 channel in mice does not impair cutaneous mechanosensation, visceral mechanonociception and hearing. J Physiol 2004;558:659-69.

24 Drew L, Rohrer DK, Price MP, et al. ASIC2 and ASIC3 do not contribute to mechanically activated currents in mammalian sensory neurons. J Physiol 2004;556:691-710.

25 Fisher BL, Schaver P. Medical and surgical options in the treatment of severe obesity. Am J Surg 2002;184:9S-16S.

26 Blackshaw LA. Receptors and transmission in the brain-gut axis: potential for novel therapies. IV. GABA(B) receptors in the brain-gastroesophageal axis. Am J Physiol Gastrointest Liver Physiol 2001;281:G311-15.

27 Lembo T, Munakata J, Mertz H, et al. Evidence for the hypersensitivity of lumbar splanchnic afferents in irritable bowel syndrome. Gastroenterology 1994; 107:1686-96

28 Munakata J, Naliboff B, Harraf F, et al. Repetitive sigmoid stimulation induces rectal hyperalgesia in patients with irritable bowel syndrome. Gastroenterology 1997;112:55-63.

29 Cervero F. Sensory innervation of the viscera: peripheral basis of visceral pain. Physiol Rev 1994;74:95-138.

30 Abo $M$, Kono T, Wang Z, et al. Impairment of gastric and jejunal myoelectrical activity during rectal distension in dogs. Dig Dis Sci 2000;45:1731-6.

31 Kreulen DL, Szurszewski JH. Reflex pathways in the abdominal prevertebral ganglia: evidence for a colo-colonic inhibitory reflex. J Physiol 1979;295:21-32 